\title{
Clickable Cellulosic Surfaces for Peptide-Based Bioassays
}

Maria Teresa Odinolfi, Alessandro Romanato, Greta Bergamaschi, Alessandro Strada, Laura Sola, Alessandro Girella, Chiara Milanese, Marcella Chiari, Alessandro Gori, Marina Cretich

Submitted date: 16/05/2019 P Posted date: 16/05/2019

Licence: CC BY-NC-ND 4.0

Citation information: Odinolfi, Maria Teresa; Romanato, Alessandro; Bergamaschi, Greta; Strada, Alessandro; Sola, Laura; Girella, Alessandro; et al. (2019): Clickable Cellulosic Surfaces for Peptide-Based Bioassays. ChemRxiv. Preprint.

The use of peptides in paper-based analytics is a highly appealing field, yet it suffers from severe limitations. This is mostly due to the loss of effective target recognition properties of this relatively small bioprobes upon nonspecific adsorption onto cellulose substrates. Here, we address this issue by introducing a simple polymer-based strategy to obtain clickable cellulosic surfaces, that we exploited for the chemoselective bioconjugation of peptide bioprobes. Our method largely outperformed standard adsorption-based immobilization strategy in a challenging, real-case immunoassay, namely the diagnostic discrimination of Zika+ individuals from healthy controls. Of note, the clickable polymeric coating not only allows efficient peptides bioconjugation, but it provides favorable anti-fouling properties to the cellulosic support. We envisage our strategy to broaden the repertoire of cellulosic materials manipulation and promote a renewed interest in peptide-based paper bioassays.

File list (2)

Clickable cellulosic surfaces for peptide-based bioassay... (647.01 KiB) view on ChemRxiv • download file Supplementary_finale.docx (24.64 MiB) view on ChemRxiv • download file 


\title{
Clickable Cellulosic Surfaces for Peptide-based Bioassays
}

\author{
Maria Teresa Odinolfi ${ }^{\mathrm{a}}$, Alessandro Romanato ${ }^{\mathrm{a}}$, Greta Bergamaschi ${ }^{\mathrm{a}}$, Alessandro Strada ${ }^{\mathrm{a}}$, Laura Sola ${ }^{\mathrm{a}}$, \\ Alessandro Girella ${ }^{\mathrm{b}}$, Chiara Milanese ${ }^{\mathrm{b}}$, Marcella Chiari ${ }^{\mathrm{a}}$, Alessandro Gori ${ }^{\mathrm{a}}$, Marina Cretich ${ }^{\mathrm{a} *}$ \\ ${ }^{a}$ : Consiglio Nazionale delle Ricerche, Istituto di Chimica del Riconoscimento Molecolare (ICRM), Via Mario Bianco, 9, \\ 20131, Milano, Italy \\ b: Pavia Hydrogen Lab, Chemistry Department, Phisical Chemistry Section, Via Taramelli 12, Pavia, Italy
}

\begin{abstract}
The use of peptides in paper-based analytics is a highly appealing field, yet it suffers from severe limitations. This is mostly due to the loss of effective target recognition properties of this relatively small probes upon nonspecific adsorption onto cellulose substrates. Here, we address this issue by introducing a simple polymer-based strategy to obtain clickable cellulose surfaces, that we exploited for the chemoselective bioconjugation of peptide bioprobes. Our method largely outperformed standard adsorption-based immobilization strategy in a challenging, real case immunoassay, namely the diagnostic discrimination of Zika+ individuals from healthy controls. Of note, the clickable polymeric coating not only allows efficient peptides bioconjugation, but it provides favorable anti-fouling properties to the cellulosic support. We envisage our strategy to broaden the repertoire of cellulosic materials manipulation and promote a renewed interest in peptide-based paper bioassays.
\end{abstract}

\section{INTRODUCTION}

Cellulosic materials are widely used substrates in the fabrication of low-cost, de-centralized, point of care tests..$^{1,3}$ Paper-based assays are indeed routinely performed to diagnose infections, to quantify glucose in urine, to test pregnancy and for blood coagulation screening. ${ }^{4.9}$ Analytical bioprobes are typically immobilized on paper by noncovalent physical adsorption, which is mainly driven by hydrophobic interactions. Despite the high loading capacity provided by cellulose tridimensional structure, binding of relatively small-sized biomolecules such as peptides may suffer from remarkable variability and can undergo desorption during iterative assay steps. Also, nonspecific adsorption of peptide ligands can mask bio-reactive functionalities resulting in decreased target binding affinities and, overall, the analytical performance can be plagued both by low reproducibility and low signal-to-background ratio. This has made peptides still underused molecular probes for paper-based immunodiagnostics, as witnessed by the very few available commercial assays relying on peptide probes. ${ }^{10}$ On the other hand, peptides and celluloses may represent a powerful partnership for applications in low resource settings conditions (e.g. prolonged storage at noncontrolled temperature and humidity, no sophisticated lab infrastructures) given advantageous features in terms of ease of handling, costs, stability. In this direction, there is a high interest in developing new enabling strategies to expand the chemical space of celluloses and prime cellulosic surfaces for covalent and spatially resolved ligands bioconjugation ${ }^{11-16}$. Of particular interest, photo-initiated thiol-yne click chemistry has been recently introduced by the Levkin group to develop micro-patterned nanocellulosic substrates. ${ }^{17}$ Yet, click-bioconjugation on cellulose materials is still an unexplored venue for the design of paperbased analytical platforms. Of note, we previously demonstrated that peptides chemoselective immobilization on microarray surfaces can boost the diagnostic performances in real-case, complex bioassays ${ }^{18,19}$ and a similar effect could be expected to improve the analytical outcomes of paper-based devices

Here we report a straightforward procedure to obtain clickable cellulosic surfaces by means of a user-friendly, polymer-based strategy, that we applied to peptide-based microarray serodiagnostics. Our approach proved effective not only to enable the covalent and chemoselective binding of peptides, but also to tailor the wettability and anti-fouling properties of the cellulosic support. We showed that both features are highly beneficial for specific antibody capturing in challenging, analytical settings, filling the existing gap in peptide-based paper assays. To this aim, we took advantage of a recently developed clickable polymer obtained by post-polymerization modification of MCP-2, a commercially available $\mathrm{N}, \mathrm{N}$-dimethylacrylamide (DMA), N-acryloyloxysuccinimide (NAS), and 3-(trimethoxysilyl)-propylmethacrylate (MAPS) copolymer ${ }^{20,21}$, using 3-azido-1-propanamine to introduce azido groups ${ }^{22}$ (Scheme 1).

\section{EXPERIMENTAL}

Nitrocellullose was coated by immersion in Copoly Azide solution $\left(1 \% \mathrm{w} / \mathrm{v}\right.$ in $\left.0.9 \mathrm{M}\left(\mathrm{NH}_{4}\right)_{2} \mathrm{SO}_{4}\right)$ for 30 minutes, rinsed with water, dried under nitrogen and cured for 15 min under vacuum at $80^{\circ} \mathrm{C}$. Full experimental details in the Supporting Information 


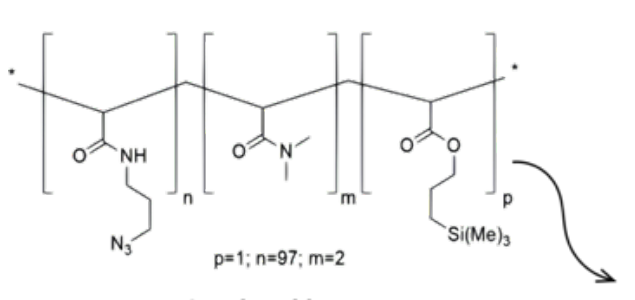

Copoly azide

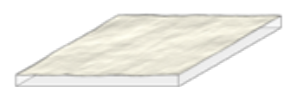

Cellulosic surface

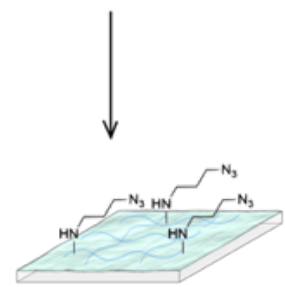

Clickable cellulose
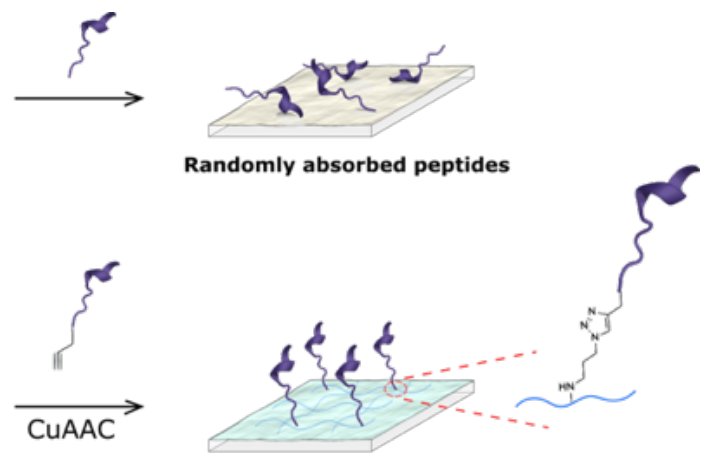

Chemoselectively bound peptides
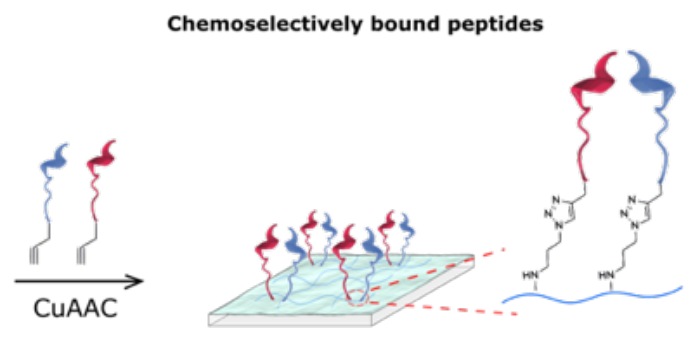

Peptides co-immobilization

P1: Prg-(O2OC) $)_{2}$-LWTDGVEESDL

P2: EMKTAEWPKSHT-(O2OC) $)_{2}$-Prg

P3: Prg-(O2OC)2-VNELPHGWKAWGKSYFVRAAKT

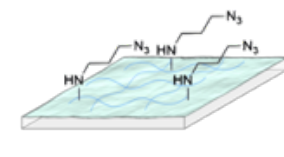

Clickable cellulose Scheme 1: cellulose modification by copoly Azide allows immobilization of yne-modified peptides via copper-catalyzed-azide-alkyne-cycloaddition
(CuAAC). In contrast to random adsorption to unmodified cellulose, the clickable cellulose allows chemoselective binding of peptides and enhanced immunoreactivity of probes by cooperative interaction resulting upon proximal peptide co-immobilization

\section{RESULTS AND DISCUSSION}

Nitrocellulose blotting membranes, a common cellulosic support for bio-analysis, was coated using a mild dip-and-rinse protocol by immersion in a polymer aqueous solution (Supporting Information). The polymer readily self-adsorbed onto the cellulosic substrate (30 minutes), without the need of surface pre-treatments. Efficient polymer adsorption was verified by FT-IR spectroscopy, showing the peculiar azide stretching band at 2100 $\mathrm{cm}-1$ (Figure 1 inset). Scanning electron microscopy (SEM) was used to compare the morphology and structure of the cellulosic surfaces. The uncoated nitrocellulose membrane is constituted by a weaving of fibers, forming elliptic micrometric cavities superimposing on different parallel planes and providing a porous structure (Figure 1-a). Moreover, sub-micron small pores can be observed along nitrocellulose fibers (indicated by the arrow). Such sub-micron porosity is instead lost upon polymeric coating, as fibers appear as covered by a layer with a melt morphology (Figure 1-b).

Interestingly, these results were duplicated using Whatman brand chromatography 1 paper (see Supplementary Information, Figure S1) suggesting applicability of the method to any cellulosic materials; however given our envisaged use of clickable paper supports for microarray immunoassays, we concentrated on the use of ready-to-use, 16-pads nitrocellulose layered on glass slides for microarrays. The wettability properties of untreated and clickable nitrocellulose were compared by static water contact angle measurements (WCAhst).

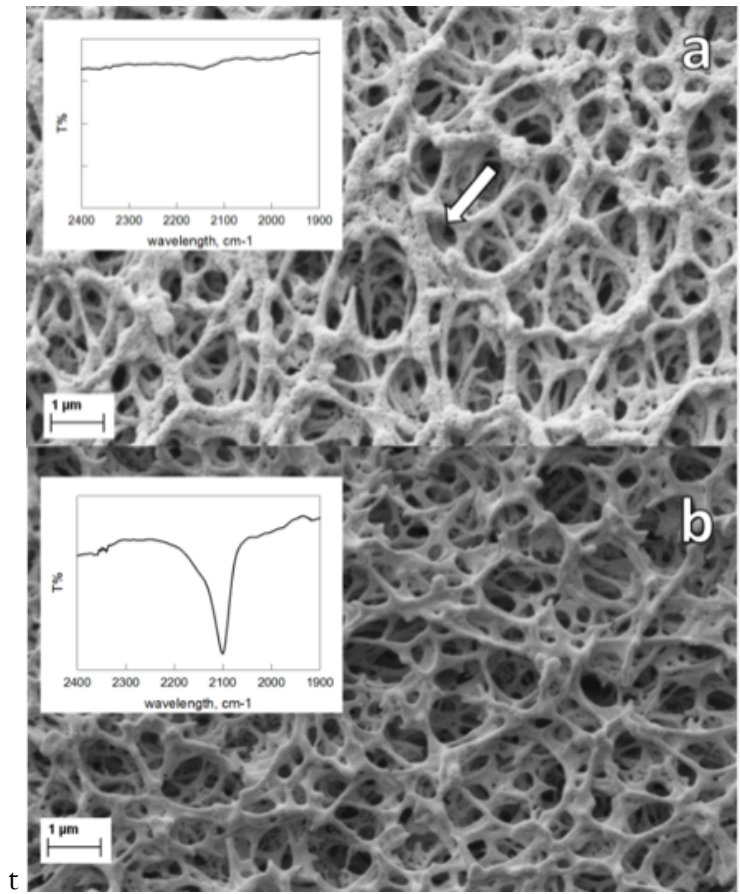

Figure 1: SEM images and FT-IR spectra (inset) of the surfaces of (a) unmodified nitrocellulose and (b) copoly Azide coated nitrocellulose (scale bar $1 \mu \mathrm{m}$ ). The distinctive azide stretching band at $2100 \mathrm{~cm}-1$ is clearly detectable on polymer treated nitrocellulose. Full FT-IR spectra of the functionalized nitrocellulose/polymer containing nitrocellulose are provided in Supplementary information. However, no major morphological alterations are visible and the high porosity of the nitrocellulose 3D structure is preserved. 
Figure 2 shows a remarkably different wettability for the unmodified and polymer-coated paper, with WCAhst values of $77.9^{\circ}$ and $19.2^{\circ}$, respectively. It is evident that the thin polymeric coating sensibly increased the surface hydrophilicity. This is in accordance with our previous findings on MCP-2 coated surface of silicon and glass. We anticipated that the observed coatinginduced hydrophilization could have an impact on the anti-fouling properties of the cellulosic surface, as the WCAhst was comparable to that of cellulose treated with BSA, commonly used to suppress the background noise. We verified the stability of the polymeric coating by repeating WCAhst measurements after prolonged exposure to water, which showed unaltered behaviour (WCAhst value of $16.4^{\circ}$ after 4 days).
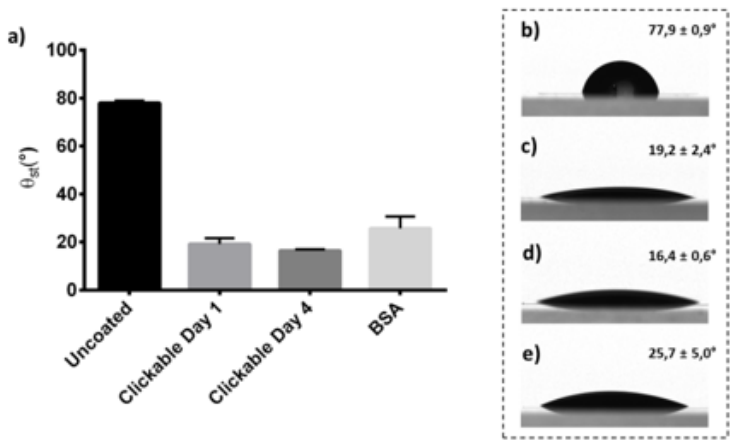

Figure 2 Water Contact Angle (WCA) measurements (a) of uncoated (b) and polymer coated nitrocellulose at day 1 (c) and after 4 days (d). Wettability after polymer coating is similar to that obtained after treatment with BSA (e).

The analytical performances of clickable and uncoated nitrocellulose were then compared in a real-case microarray immunoassay to reveal arbovirus infections by detection of antibodies directed against the Zika viral antigen NS1. To validate our platform, we selected a small panel of known NS1-antigen-derived immunoreactive peptides (Scheme 1). While peptide P1 is reactive as single molecular entity, we previously demonstrated that the effective discrimination of Zika positive individuals by peptides P2 and P3 relies on their cooperative interaction resulting upon spatially proximal co-immobilization (Scheme 1). ${ }^{23}$ In this latter and more demanding case, a fine control of peptidic probes display inducing a cooperative binding mechanism is crucial to efficiently capture Zika virus antibodies. To enable click bioconjugation onto azido-modified nitrocellulose, P1-P3 peptides were modified with a short-chain PEG spacer $(\mathrm{O} 2 \mathrm{Oc})$ including a terminal alkyne moiety. Peptides were arrayed by a piezoelectric spotter, either dissolved in PBS to allow spontaneous adsorption on uncoated nitrocellulose or reacted via CuACC to provide chemoselective covalent binding (See Supporting Information). To suppress nonspecific interactions during the assays, untreated nitrocelluloses were preliminary incubated with a $2 \% \mathrm{w} / \mathrm{v}$ BSA solution, whereas azido-modified surfaces, due to the expected favorable anti-fouling properties provided by the polymeric coating, were simply washed in a 2 mM EDTA aqueous solution. The two 16-pads nitrocellulose slides were incubated with Zika positive human sera and captured antibodies were detected using an alkaline phosphatase (AP) labelled secondary antibody to provide colorimetric quantification of peptide immune-recognition, using nitro blue tetrazolium/5-bromo-4-chloro-3-indolyl phosphate (NBT/BCIP). We could immediately assess a striking difference on the extent of captured antibodies between clickable and uncoated nitrocellulose. While for untreated nitrocellulose colored spots were indeed barely detectable and inhomogeneous, i.e. highlighting poor immune recognition, the chemoselectively bound probes were efficiently recognized giving rise to dark-blue colored regular spots (Figure 3-a). This is mostly indicative that peptides affinity to their target antibodies is strongly compromised by a nonspecific strategy of immobilization, where immune-reactive regions are sacrificed upon surface adsorption. This may be particularly relevant in all of those demanding assays that do not rely on an exquisite affinity of the immobilized bioprobe for the target antibody. We then confirmed that the preserved immunoreactivity of P1, P2, P3 probes on the clickable nitrocellulose support could be translated into efficient discrimination between Zika positive individuals and healthy controls (Figure 3-b). As we previously observed, the combined presentation of peptides P2 and P3 (probe C) proved the most effective in providing statistically relevant discrimination between the two groups with $\mathrm{p}<0.01$ (Figure 3-c).

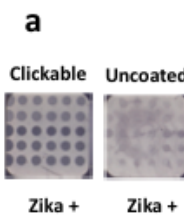

$$
\text { b }
$$
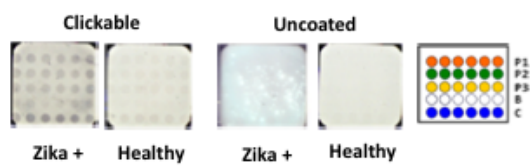

Zika + Healthy
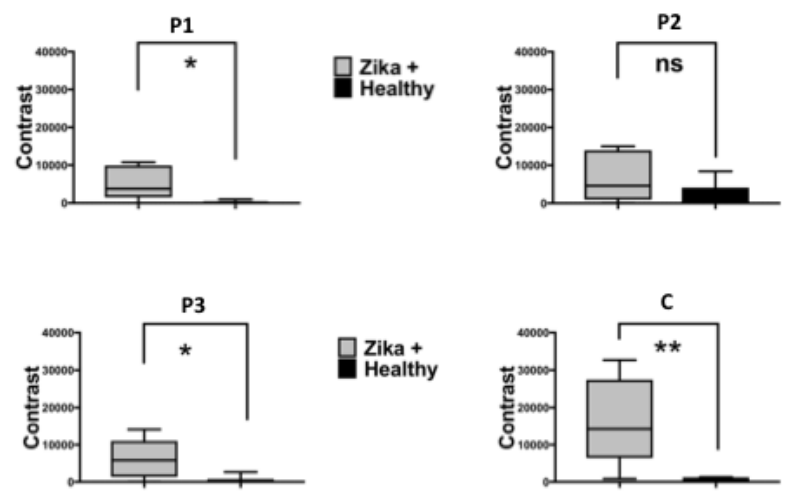

Figure 3. a): representative clickable and uncoated nitrocellulose pads spotted with peptide arrays after incubation with a Zika positive sample, dark-blue regular spots are detectable only on polymer coated pads. b): representative coated and uncoated nitrocellulose pads spotted with peptide arrays (see spotting scheme on the right) after incubation with a Zika positive sample and an healthy control, discrimination between infected and healthy serum is only possible on clickable nitrocellulose. c): Scatter plots reporting mean immunoreactivity with SD of Zika positive and healthy control individuals and results of the unpaired $t$ Test Zika infection diagnosis probe P1, P2, P3 and C (combination of peptides P2 and P3). ns = not significant. Significant: $\mathrm{p}<0.05 ;^{*}=\mathrm{p}<0.05 ;^{* *}=\mathrm{p}<0.01$. Photographs of whole 16-pad microarray slides available in the Supporting Information (Figure S3 and S4)

This underlines the possibility to finely control peptidic probes surface display even on cellulosic supports, which is, to the best of our knowledge, an unprecedented result in paperbased bioassays. It is also worth underlining that, in the same assay, an alternative oriented bioconjugation strategy based on biotinylated peptides and streptavidin functionalized 
nitrocellulose resulted in poor immunoreactivity and inconsistent discrimination between healthy and infected samples (Supporting Information, Figure S5). These results are worth of particular consideration as celluloses are rather complex substrates, mostly due to peculiar porosity properties and related high surface-to-volume ratio that can amplify interface effects such as nonspecific interactions and the formation of interfering multiple bioprobe layers.

\section{CONCLUSIONS}

Here, we move beyond current limitations of paper-based bioassays by introducing an easy and user-friendly method for peptide chemoselective bioconjugation onto nitrocellulose supports based on a clickable polymeric coating. We demonstrate that our strategy is suitable for peptide probes stable binding while avoiding to compromise their immunoreactivity to target antibodies, since nonspecific interactions with the nitrocellulose support are abolished. The structural properties of nitrocellulose are maintained, but the wettability and anti-fouling properties provided by the polymeric coating are effective in suppressing background noise signal even when complex biological samples, such as sera (see Supplementary Information Figure S6). We demonstrate that these favorable features can be exploited to perform sophisticated molecular bioassays, in this case the serological discrimination between Zika positive individuals and healthy controls, using a peptide-based assay. Overall, our results enrich the toolbox for the controlled manipulation of cellulosic material interfacial properties, broadening their compatibility with smallsized peptide molecular probes.

\section{ASSOCIATED CONTENT}

\section{Supporting Information}

Full experimental details and additional Figures are available in the Supporting Information file

\section{AUTHOR INFORMATION}

\section{Corresponding Authors}

*Email: marina.cretich@icrm.cnr.it ; $\underline{\text { alessandro.gori@icrm.cnr.it }}$

\section{ACKNOWLEDGMENT}

Work partially funded by Regione Lombardia, project READY (Regional Network for developing diagnostic methods in rapid response to emerging epidemics and bio-emergencies) ID 229472.

\section{REFERENCES}

(1) López-Marzo, A. M.; Merkoçi, A. Paper-Based Sensors and Assays: A Success of the Engineering Design and the Convergence of Knowledge Areas. Lab Chip 2016, 16 (17), 3150-3176. https://doi.org/10.1039/C6LC00737F.

(2) Parolo, C.; Merkoçi, A. Paper-Based Nanobiosensors for Diagnostics. Chemical Society Reviews. 2013, pp 450-457. https://doi.org/10.1039/c2cs35255a.

(3) Klemm, D.; Heublein, B.; Fink, H.-P.; Bohn, A. Cellulose: Fascinating Biopolymer and Sustainable Raw Material. Angew. Chemie Int. Ed. 2005, 44 (22), 3358-3393. https://doi.org/10.1002/anie.200460587.
(4) Martinez, A. W.; Phillips, S. T.; Whitesides, G. M.; Carrilho, E. Diagnostics for the Developing World: Microfluidic Paper-Based Analytical Devices. Anal. Chem. 2010, 82 (1), 3-10. https://doi.org/10.1021/ac9013989.

(5) Quesada-González, D.; Merkoçi, A. Nanoparticle-Based Lateral Flow Biosensors. Biosens. Bioelectron. 2015, 73, 47-63. https://doi.org/10.1016/j.bios.2015.05.050.

(6) Singh, A. T.; Lantigua, D.; Meka, A.; Taing, S.; Pandher, M.; Camci-Unal, G. Paper-Based Sensors: Emerging Themes and Applica$\begin{array}{lll}\text { tions. } & \text { Sensors } & \text { (Switzerland). }\end{array}$ https://doi.org/10.3390/s18092838.

(7) Hu, J.; Zhang, Z.-L.; Wen, C.-Y.; Tang, M.; Wu, L.-L.; Liu, C.; Zhu, L.; Pang, D.-W. Sensitive and Quantitative Detection of C-Reaction Protein Based on Immunofluorescent Nanospheres Coupled with Lateral Flow Test Strip. Anal. Chem. 2016, 88 (12), 6577-6584. https://doi.org/10.1021/acs.analchem.6b01427.

(8) Morales-Narváez, E.; Naghdi, T.; Zor, E.; Merkoçi, A. Photoluminescent Lateral-Flow Immunoassay Revealed by Graphene Oxide: Highly Sensitive Paper-Based Pathogen Detection. Anal. Chem. 2015, 87 (16), 8573-8577. https://doi.org/10.1021/acs.analchem.5b02383.

(9) Li, H.; Steckl, A. J. Paper Microfluidics for Point-of-Care Blood-Based Analysis and Diagnostics. Anal. Chem. 2019, 91 (1), $352-$ 371. https://doi.org/10.1021/acs.analchem.8b03636.

(10) de Puig, H.; Bosch, I.; Gehrke, L.; Hamad-Schifferli, K. Challenges of the Nano-Bio Interface in Lateral Flow and Dipstick Immunoassays. Trends in Biotechnology. 2017, pp 1169-1180. https://doi.org/10.1016/j.tibtech.2017.09.001.

(11) Kong, F.; Hu, Y. F. Biomolecule Immobilization Techniques for Bioactive Paper Fabrication. Anal. Bioanal. Chem. 2012, 403 (1), 713. https://doi.org/10.1007/s00216-012-5821-1.

(12) Riccardi, C.; McCormick, S.; Kasi, R.; Kumar, C. A Modular Approach for Interlocking Enzymes in Whatman Paper. Angew. Chemie 2018, $\quad 130 \quad$ (32),

https://doi.org/10.1002/ange.201805074.

(13) Deraney, R. N.; Mace, C. R.; Rolland, J. P.; Schonhorn, J. E. Multiplexed, Patterned-Paper Immunoassay for Detection of Malaria and Dengue Fever. Anal. Chem. 2016, 88 (12), 6161-6165. https://doi.org/10.1021/acs.analchem.6b00854.

(14) Große, S.; Wilke, P.; Börner, H. G. Easy Access to Functional Patterns on Cellulose Paper by Combining Laser Printing and MaterialSpecific Peptide Adsorption. Angew. Chemie Int. Ed. 2016, 55 (37), 11266-11270. https://doi.org/10.1002/anie.201601603.

(15) Draz, M. S.; Moazeni, M.; Venkataramani, M.; Lakshminarayanan, H.; Saygili, E.; Lakshminaraasimulu, N. K.; Kochehbyoki, K. M.; Kanakasabapathy, M. K.; Shabahang, S.; Vasan, A.; et al. Hybrid PaperPlastic Microchip for Flexible and High-Performance Point-of-Care Diagnostics. Adv. Funct. Mater. 2018, 28 (26), 1707161. https://doi.org/10.1002/adfm.201707161.

(16) Dong, Y.; Chen, H.; Zhang, S.; Wang, P.; Lu, X.; Liu, W.; Wu, Z. "Click-Chemical" Modification of Cellulose Acetate Nanofibers: A Versatile Platform for Biofunctionalization. J. Mater. Chem. B 2018, 6 (28), 4579-4582. https://doi.org/10.1039/c8tb01401a.

(17) Guo, J.; Filpponen, I.; Johansson, L.-S.; Heißler, S.; Li, L.; Levkin, P.; Rojas, O. J. Micro-Patterns on Nanocellulose Films and Paper by Photo-Induced Thiol-yne Click Coupling: A Facile Method toward Wetting with Spatial Resolution. Cellulose 2018, 25 (1), 367-375. https://doi.org/10.1007/s10570-017-1593-2.

(18) Gori, A.; Sola, L.; Gagni, P.; Bruni, G.; Liprino, M.; Peri, C.; Colombo, G.; Cretich, M.; Chiari, M. Screening Complex Biological Samples with Peptide Microarrays: The Favorable Impact of Probe Orientation via Chemoselective Immobilization Strategies on Clickable Polymeric Coatings. Bioconjug. Chem. 2016, 27 (11), 2669-2677. https://doi.org/10.1021/acs.bioconjchem.6b00426.

(19) Gori, A.; Cretich, M.; Vanna, R.; Sola, L.; Gagni, P.; Bruni, G.; Liprino, M.; Gramatica, F.; Burastero, S.; Chiari, M. Multiple Epitope Presentation and Surface Density Control Enabled by Chemoselective Immobilization Lead to Enhanced Performance in IgE-Binding 
Fingerprinting on Peptide Microarrays. Anal. Chim. Acta 2017. https://doi.org/10.1016/j.aca.2017.06.027.

(20) Pirri, G.; Damin, F.; Chiari, M.; Bontempi, E.; Depero, L. E. Characterization of A Polymeric Adsorbed Coating for DNA Microarray Glass Slides. Anal. Chem. 2004, 76 (5), 1352-1358. https://doi.org/10.1021/ac0352629.

(21) Cretich, M.; Di Carlo, G.; Longhi, R.; Gotti, C.; Spinella, N.; Coffa, S.; Galati, C.; Renna, L.; Chiari, M. High Sensitivity Protein Assays on Microarray Silicon Slides. Anal. Chem. 2009, 81 (13). https://doi.org/10.1021/ac900658c.
(22) Sola, L.; Damin, F.; Gagni, P.; Consonni, R.; Chiari, M. Synthesis of Clickable Coating Polymers by Postpolymerization Modification: Applications in Microarray Technology. Langmuir 2016, 32 (40), 10284-10295. https://doi.org/10.1021/acs.langmuir.6b02816.

(23) Sola, L.; Gagni, P.; D’Annessa, I.; Capelli, R.; Bertino, C.; Romanato, A.; Damin, F.; Bergamaschi, G.; Marchisio, E.; Cuzzocrea, A.; et al. Enhancing Antibody Serodiagnosis Using a Controlled Peptide Coimmobilization Strategy. ACS Infect. Dis. 2018, 4 (6), 998-1006. https://doi.org/10.1021/acsinfecdis.8b00014. 
Clickable cellulosic surfaces for peptide-based bioassay... (647.01 KiB) view on ChemRxiv • download file 


\section{Supplementary Information}

\section{Clickable cellulosic surfaces for peptide-based bioassays}

Maria Teresa Odinolfi, ${ }^{[a]}$ Alessandro Romanato, ${ }^{[a]}$ Greta Bergamaschi, ${ }^{\text {[a] }}$ Alessandro Strada ${ }^{[a]}$ Laura Sola, ${ }^{[a]}$ Alessandro Girella, ${ }^{[b]}$ Chiara Milanese, ${ }^{[b]}$ Marcella Chiari, ${ }^{[a]}$ Alessandro Gori ${ }^{[a]}$, Marina Cretich*[a]

Index:

Materials and Methods Page S1

Figure S1: SEM images Page S4

Figure S2 : FT-IR spectra Page S4

Figure S3 : photograph of nitrocellulose slides (immunoreactivity) Page S5

Figure S4 : photograph of nitrocellulose slides (patients vs controls) Page S5

Figure S5 : photograph of nitrocellulose slide (biotin-streptavidin immobilization) Page S6

Figure S6 : photograph of nitrocellulose pads (background) Page S6 


\section{Materials and Methods}

\section{Reagents}

Reagents for peptide synthesis were from Iris Biotech (Marktredwitz, Germany). Other chemicals were from Sigma-Aldrich (St. Louis, MO, USA) if not stated otherwise. All solvents for solid-phase peptide synthesis (SPPS) were used without further purification. HPLC grade acetonitrile (ACN) and ultrapure 18.2 $\Omega$ water (Millipore-MilliQ) were used for the preparation of all buffers for liquid chromatography. The chromatographic columns were from Phenomenex (Torrance CA, USA). HPLC eluent A: 97.5\% H2O, 2.5\% ACN, 0.7\%TFA; HPLC eluent B: 30\% H2O, 70\% ACN, 0.7\%TFA. Alkaline Phosphatase-conjugated AffiniPure F(ab')2 Fragment Rabbit Anti-Human IgG was obtained from Jackson Immunoresearch (West Grove, PA, USA). LowCross-Buffer was from Candor (Wangen, Germany).

Copoly Azide was obtained from MCP-2 (Lucidant Polymers, Sunnyvale CA) by postpolymerization modification reaction with 3-azido-1-propanamine as reported in [L. Sola, F. Damin, P. Gagni, R. Consonni, M. Chiari, Synthesis of Clickable Coating Polymers by Postpolymerization Modification: Applications in Microarray Technology, Langmuir. 32 (2016)]

\section{Peptide Synthesis}

Peptides p1-p3 were prepared and characterized as previously reported [L. Sola, P. Gagni, I. D’Annessa, R. Capelli, C. Bertino, A. Romanato, F. Damin, G. Bergamaschi, E. Marchisio, A. Cuzzocrea, et al., ACS Infect. Dis. 2018, 4, 998-1006]. Briefly, all peptides were synthesized by stepwise microwave-assisted Fmoc-SPPS on a Biotage ALSTRA Initiator+ peptide synthesizer according to Fmoc-based protocols. Sequences were assembled on a 2-CTC resin and chain elongation was performed by iterative cycles of amino acids coupling ( 1 x 20min, $50 \mathrm{C}$; Oxyma/DIC as activators) and Fmoc-deprotection using a $20 \%$ piperidine solution in DMF (1x10min). Upon complete chain assembly, peptides were cleaved from the resin using a $2.5 \% \mathrm{TIS}, 2.5 \%$ thioanisole, $2.5 \%$ water, $92.5 \%$ TFA mixture. Crude peptides were then purified by preparative RPHPLC. MS analysis was performed separately on purified material.

\section{Samples}

A panel of positive ZIKV serum samples was purchased from Boca Biolistics (Pompano Beach, $\mathrm{FL}$ ). Samples were proved positive by MAC Elisa test performed by the purchaser and confirmed positive for anti-Zika lgG by an NS1 based ELISA test (ZIKV IgG from Dia.Pro. (Sesto San Giovanni, Italy) according to manufacturer instructions. Human sera from healthy donors used in the study were obtained from voluntaries, which donated blood for transfusion purposes with the 
collaboration of Transfusion Centre (IRCCS Ca' Granda Policlinico Ospedale Maggiore in Milan, Italy) and their use was permitted for research purposes only. Both donor and recipient's identities are protected by the privacy laws governing the Italian Transfusion System. The purpose of production of blood products is only of the donor's free will and with the latter's consent.

\section{Nitrocellulose and Whatman paper coating}

Nitrocellullose slides (Fast Slide 16-Pad, Whatman®), nitrocellulose blotting membranes (BioTrace NT Blotting membranes from PALL), Whatman filter paper 1, were coated by Copoly Azide by immersion in a polymer solution $\left(1 \% \mathrm{w} / \mathrm{v}\right.$ in $\left.0.9 \mathrm{M}\left(\mathrm{NH}_{4}\right)_{2} \mathrm{SO}_{4}\right)$ for 30 minutes, then rinsed with water, dried under nitrogen and cured for $15 \mathrm{~min}$ under vacuum at $80^{\circ} \mathrm{C}$. Blotting membranes were used for the FT-IR measurements and for the SEM microscopy experiments. 16-Pad Slides were used for the contact angle measurements and the bioassays

\section{Peptide microarrays}

Peptides were first dissolved in DMSO to $1 \mathrm{mM}$ stock solution and then diluted to the final spotting concentration $(100 \mu \mathrm{M})$ into the printing buffer for uncoated slides (PBS) or for CuACC conjugation on Copoly Azide coated surfaces (25 mM Na/Acetate pH 4.8, 15 mM Trehalose, $100 \mu \mathrm{M}$ CuSO4, $400 \mu \mathrm{M}$ THPTA and $6.25 \mathrm{mM}$ Ascorbic Acid). Microarrays were arrayed using a non-contact S12

Spotter (Scienion Co., Berlin, Germany), depositing 5 drops for each spot. All the peptides samples were printed in replicates. Up to sixteen arrays were spotted on the slides, corresponding to the sixteen compartments created by NEXTERION* IC-16 sixteen well Incubation Chamber from Schott (Jena, Germany). The same protocol was applied to nitrocellulose uncoated slides.

Printed slides were placed in a humid chamber and incubated overnight at room temperature. The following day the slides coated with Copoly Azide were immersed in 2 mM EDTA water solution for $1 \mathrm{~h}$, while uncoated slides were immersed in a solution of $1 \%$ BSA in PBS. Finally, the slides were washed with water and dried under a stream of nitrogen.

\section{Bioassay}

Sera were diluted 3:50 v/v in LowCross-Buffer and $50 \mu \mathrm{L}$ of sample were added into each microarray well, incubated for 30 minutes on a shaker $\left(70 \mathrm{rpm}, 22^{\circ} \mathrm{C}\right)$. ZIKV positive and healthy sera were incubated in parallel app to 16 samples per slide. Negative controls included blank arrays incubated only with the incubation buffer. The microarray slides were then rinsed for 15 minutes with washing buffer $(0.05 \mathrm{M}$ Tris/ $\mathrm{HCl} \mathrm{pH} 9,0.25 \mathrm{M} \mathrm{NaCl}, 0.05 \% \mathrm{v} / \mathrm{v}$ Tween 20) and incubated statically with $50 \mu \mathrm{L}$ of Alkaline Phosphatase-conjugated AffiniPure $\mathrm{F}\left(\mathrm{ab}{ }^{\prime}\right)_{2}$ Fragment Rabbit Anti-Human IgG 1mg/mL diluted 1:300 in LowCross-Buffer for 1 hour. The slides were 
washed with PBS for 5 minutes and then with washing buffer for further 5 minutes. For colorimetric development, the slides were incubated with BCIP/NBT (Blue Liquid Substrate System for Membranes) from Sigma for 10 minutes in static manner, then washed with water and dried under nitrogen.

\section{Detection}

The results were visible by naked eye. Pictures of the arrays were taken using a stereomicroscope equipped with a Nikon SMZ 1500 camera and the Infinity Capture software from Lumenera Corporation. ImageJ software was used to analyse the images as follows: luminosities of spots and background were evaluated through the "Measure" function designing circular areas whose colour intensity was calculated by subtracting the background around the spots. The results were expressed as the absolute values of the negative contrast.

\section{Material characterization}

Infrared spectra of paper strips were recorded using a Nicolet Nexus FTIR spectrometer equipment with U-ATR device from 400 to $4,000 \mathrm{~cm}-1$. For each sample, spectra were collected (128 scans) using solid FT-IR support. The morphology of the gold sputtered materials was fully characterized using a EvoMA10 (Zeiss, Oberkochen, Germany) Scanning Electron Microscope (SEM) equipped with a LaB6 filament. The wettability properties of untreated and clickable nitrocellulose were evaluated by static water contact angle measurements using a KSV Instruments Contact Angle equipped with a CAM 200 camera. 


\section{Supplementary Figures}

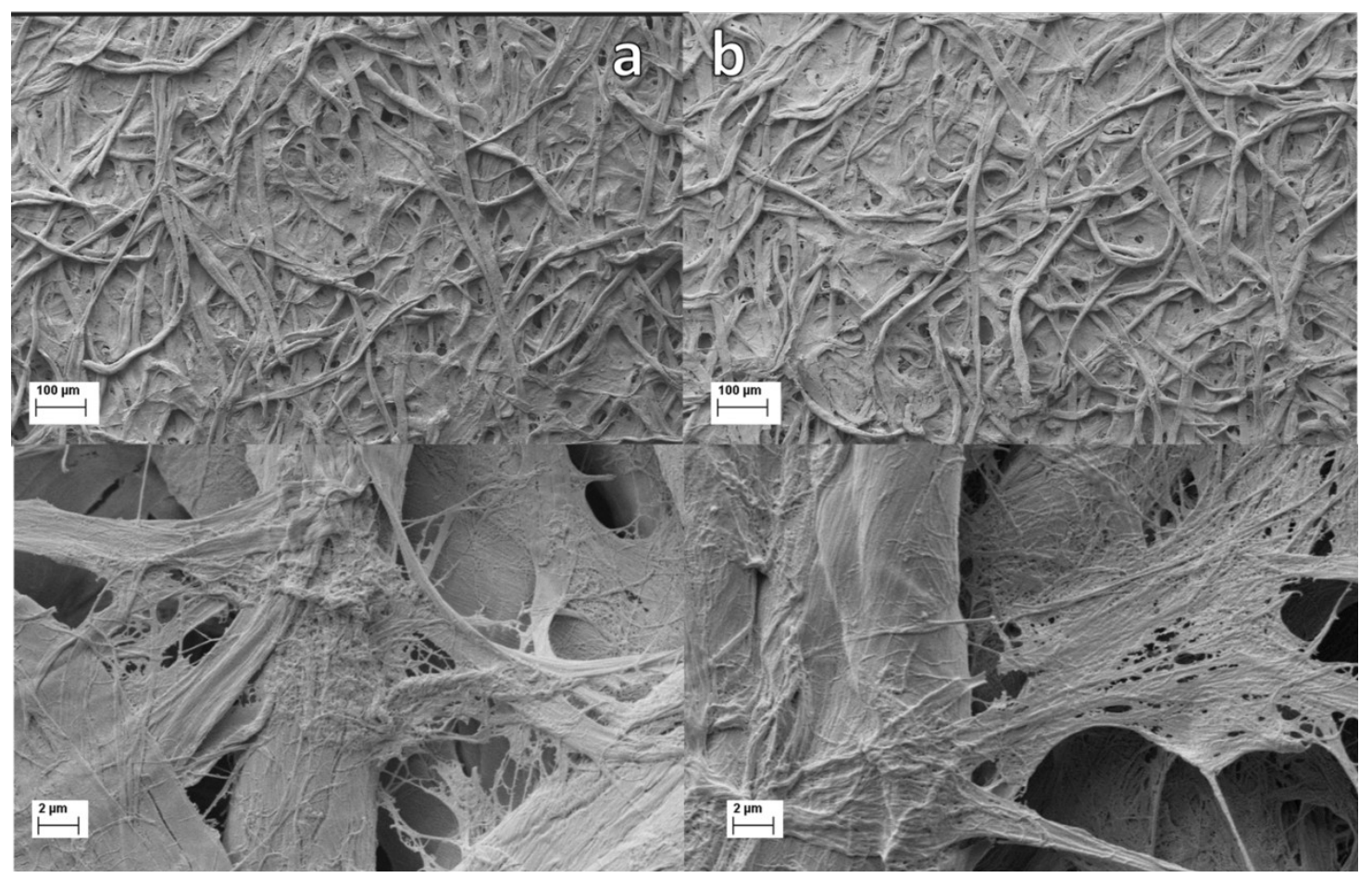

Figure S1: SEM images of the surfaces (a) unmodified b) polymer-coated Whatman filter paper (scale bar $1 \mu \mathrm{m}$ ).

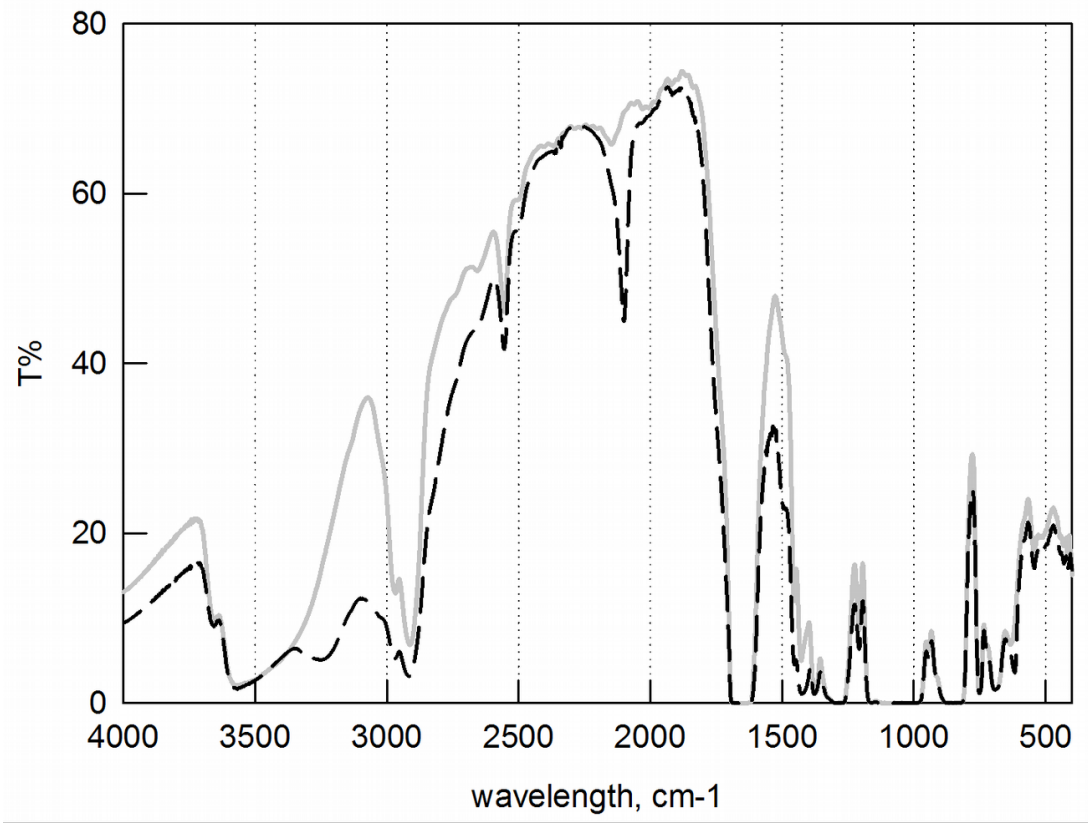

Figure S2: FT-IR spectra of the functionalized nitrocellulose/polymer containing nitrocellulose (black dashed line) and the uncoated nitrocellulose (grey solid line). The band at $2100 \mathrm{~cm}^{-1}$ is assigned to azide group stretching. 


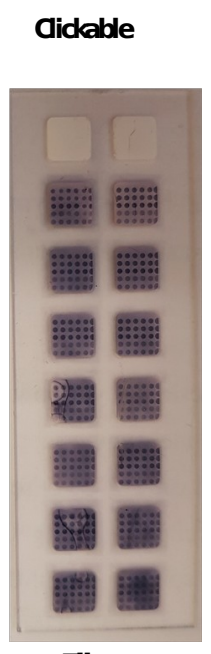

Zka +

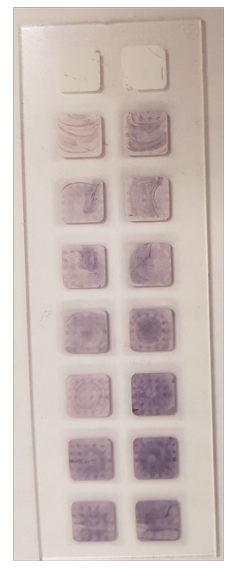

Zlka+

Figure S3: photographs of 16 pad nitrocellulose slides incubated with 14 Zika positive serum samples on copoly Azide modified surface (left) and on uncoated support (right). Immunoreactivity of peptide spots is much higher on the clickable surface. Peptide spots on the uncoated slide are barely visible. Colorimetric reaction was prolonged on both slides to enhance the visibility of peptide spots on the uncoated nitrocellulose.

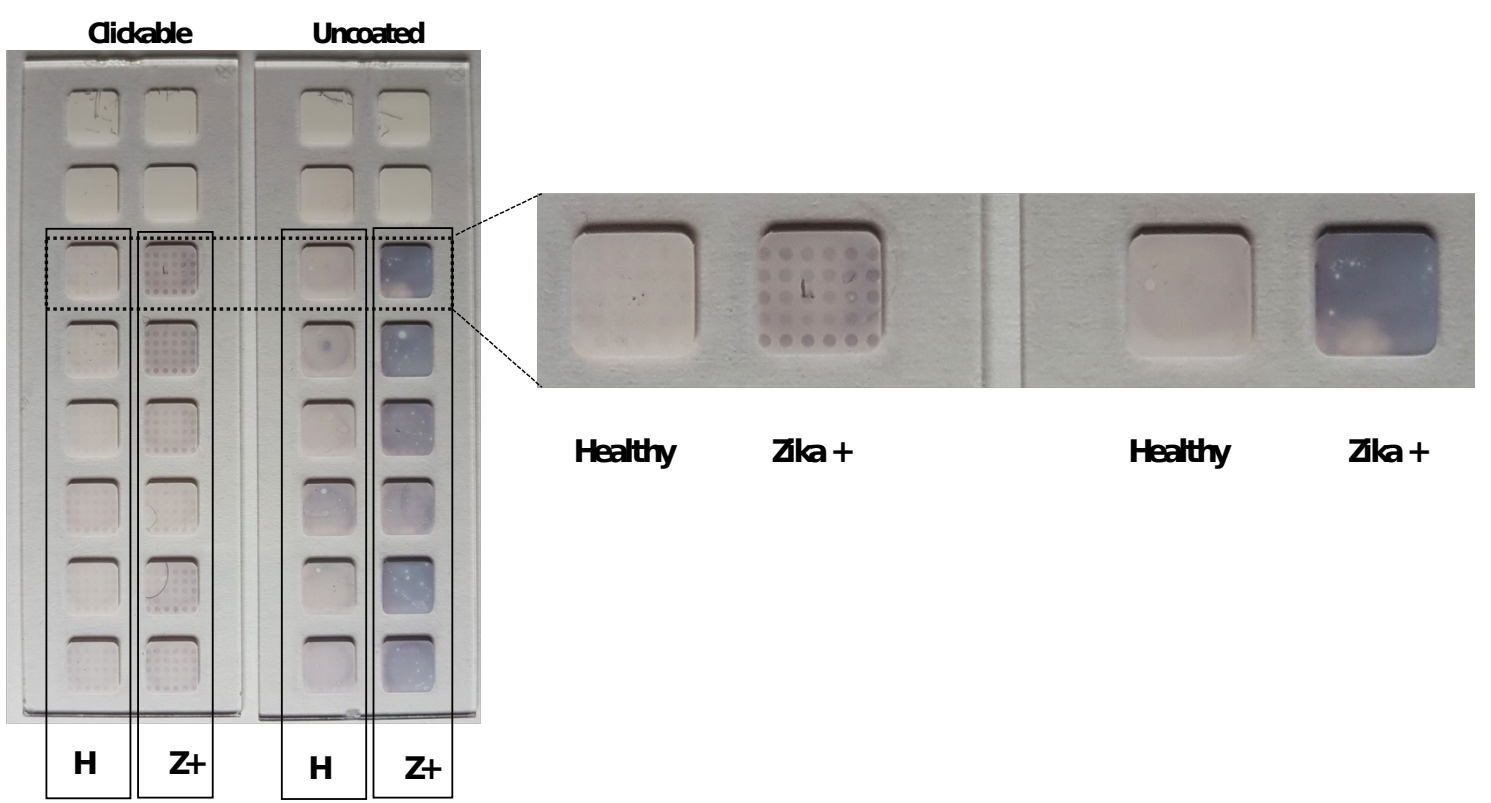

Figure S4: photographs of 16 pad nitrocellulose slides incubated with 6 healthy control sera (columns $\mathrm{H}$ ) and 6 Zika positive serum samples (column Z+) on copoly Azide modified surface (left slide) and on uncoated support (right slide). Slides were used in parallel using the same bioassay conditions. Colorimetric reaction with enzyme substrates BCIP/NBT was standardized at 5 minutes for all surfaces and samples. On clickable surface, for all analysed samples, the Zika positive sera provided distinguishable coloured spots of much higher intensity in comparison to that provided by the healthy controls. On the contrary, on uncoated nitrocellulose, in the same conditions of assay and colorimetric reaction, no spots were distinguishable from background. 


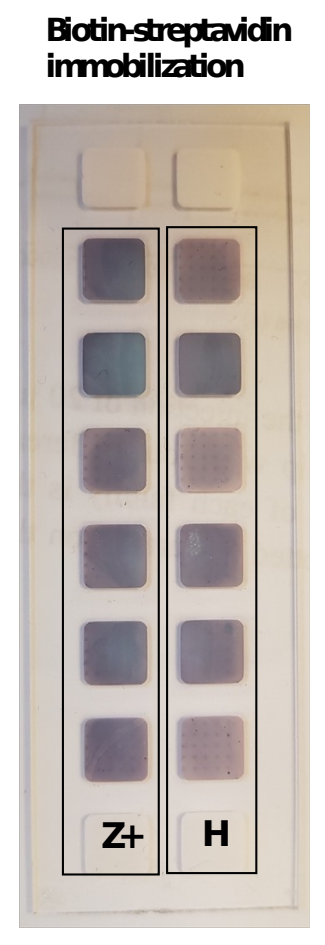

Figure S5: uncoated 16 pads nitrocellulose slide co-spotted with 3 droplets streptavidin $(1 \mathrm{mg} / \mathrm{mL})$ and 3 droplets of 50 uM biotinylated peptides. Spots are barely visible for some of the samples and no discrimination between healthy and positive sera is feasible

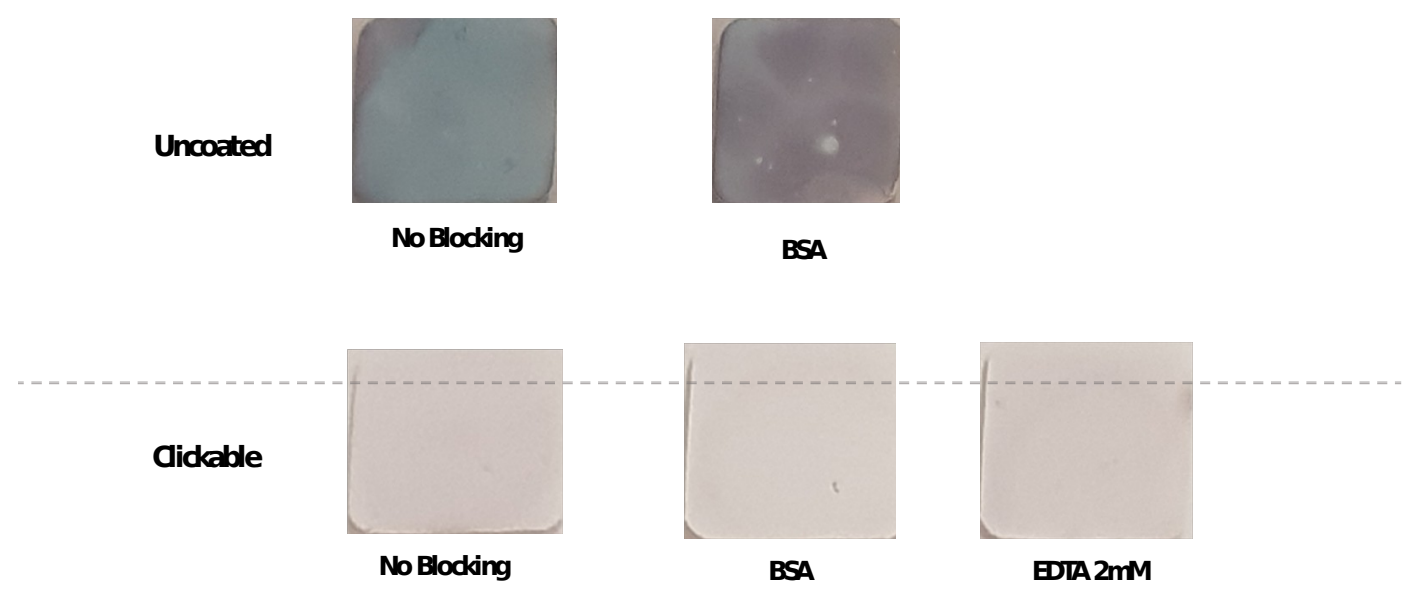

Figure S6: comparison of background signal nitrocellulose pads after incubation with serum and Alkaline Phosphatase-conjugated AffiniPure $\mathrm{F}\left(\mathrm{ab}^{\prime}\right)_{2}$ Fragment Rabbit Anti-Human IgG. Colorimetric reaction with enzyme substrates $\mathrm{BCIP/NBT}$ was standardized at 5 minutes for all surfaces revealing differences in background among the tested surfaces. The uncoated nitrocellulose provided the highest background, even when surface was blocked by BSA. The clickable (copoly Azide modified) nitrocellulose showed the lowest background. Notably, background is not affected by blocking or washing, demonstrating the inherent anti-fouling propertied of the copoly Azide coating. 
